\title{
Competition Sensing Changes Antibiotic Production in Streptomyces
}

\author{
(D) Sanne Westhoff, a (D) Alexander M. Kloosterman, ${ }^{a}$ Stephan F. A. van Hoesel, ${ }^{a}$ (D) Gilles P. van Wezel, ${ }^{a, b}$ (D) Daniel E. Rozen ${ }^{a}$
}

alnstitute of Biology, Leiden University, Leiden, The Netherlands

${ }^{b}$ Netherlands Institute of Ecology (NIOO-KNAW), Wageningen, The Netherlands

ABSTRACT One of the most important ways that bacteria compete for resources and space is by producing antibiotics that inhibit competitors. Because antibiotic production is costly, the biosynthetic gene clusters coordinating their synthesis are under strict regulatory control and often require "elicitors" to induce expression, including cues from competing strains. Although these cues are common, they are not produced by all competitors, and so the phenotypes causing induction remain unknown. By studying interactions between 24 antibiotic-producing strains of streptomycetes, we show that strains commonly inhibit each other's growth and that this occurs more frequently if strains are closely related. Next, we show that antibiotic production is more likely to be induced by cues from strains that are closely related or that share secondary metabolite biosynthetic gene clusters (BGCs). Unexpectedly, antibiotic production is less likely to be induced by competitors that inhibit the growth of a focal strain, indicating that cell damage is not a general cue for induction. In addition to induction, antibiotic production often decreases in the presence of a competitor, although this response was not associated with genetic relatedness or overlap in BGCs. Finally, we show that resource limitation increases the chance that antibiotic production declines during competition. Our results reveal the importance of social cues and resource availability in the dynamics of interference competition in streptomycetes.

IMPORTANCE Bacteria secrete antibiotics to inhibit their competitors, but the presence of competitors can determine whether these toxins are produced. Here, we study the role of the competitive and resource environment on antibiotic production in Streptomyces, bacteria renowned for their production of antibiotics. We show that Streptomyces cells are more likely to produce antibiotics when grown with competitors that are closely related or that share biosynthetic pathways for secondary metabolites, but not when they are threatened by competitor's toxins, in contrast to predictions of the competition sensing hypothesis. Streptomyces cells also often reduce their output of antibiotics when grown with competitors, especially under nutrient limitation. Our findings highlight that interactions between the social and resource environments strongly regulate antibiotic production in these medicinally important bacteria.

KEYWORDS Streptomyces, antibiotic production, interference competition, microbial ecology, social microbiology

Dacteria live in diverse communities, where they compete with other microbes for resources and space. Competition between different species can be regulated by the differential uptake and use of specific nutrients. It can also be driven by secreted toxins, like antibiotics or bacteriocins, that kill or inhibit competitors. Antibiotics and bacteriocins can allow producing strains to invade established habitats or repel invasion by other strains $(1,2)$. However, these compounds are expected to be metabolically expensive to make and so should only be produced against genuine threats from competitors. The competition sensing hypothesis predicts that microbes should

Citation Westhoff S, Kloosterman AM, van Hoesel SFA, van Wezel GP, Rozen DE. 2021. Competition sensing changes antibiotic production in Streptomyces. mBio 12:e272920. https://doi.org/10.1128/mBio.02729-20. Editor Gerard D. Wright, McMaster University Copyright $\odot 2021$ Westhoff et al. This is an open-access article distributed under the terms of the Creative Commons Attribution 4.0 International license.

Address correspondence to Sanne Westhoff, s.westhoff@biology.leidenuniv.nl, or Daniel E. Rozen, d.e.rozen@biology.leidenuniv.nl.

Received 24 September 2020

Accepted 14 December 2020

Published 9 February 2021 
upregulate toxin production when they experience cell damage or nutrient limitation caused by competitors (3). Alternatively, bacteria can also sense competitors by detecting secreted signals that predict imminent danger, but that cause no direct harm themselves: for example, small molecule or peptide signals that are used to regulate toxin production by quorum sensing (3). Consistent with the predictions of competition sensing, several studies have observed that microbes facultatively increase antibiotic or bacteriocin production when they are grown in coculture with a competing strain (4-7). However, bacteria do not respond to all competitors in this way $(7,8)$. Moreover, counter to predictions of competition sensing, cocultivation can also cause strains to reduce antibiotic production $(4,6)$, rather than to respond aggressively to provocation. Why do bacteria respond to some competitors with aggression, but not to others? Similarly, are some cues from competitors more likely to elicit responses than others? To date, the answers to these questions have remained unknown. This shortcoming limits our ability to identify and induce cryptic antibiotic gene clusters for drug discovery and prevents a detailed understanding of the factors regulating the competitive dynamics of bacterial populations.

The aim of this article is to address these issues in the context of bacteria from the prolific antibiotic-producing family Streptomycetaceae (9). These filamentous, sporeforming bacteria are renowned for their production of secondary metabolites, including many clinically useful antibiotics, antihelminthic agents, and anticancer drugs (10). Antibiotic production in streptomycetes is associated with the developmental stage of the colony and typically coincides with the onset of sporulation $(11,12)$. We refer to this type of autonomous production as "constitutive" because it occurs in the absence of influence from other species. In addition, we and others have found that the presence of other strains in coculture can alter antibiotic production by increasing or reducing antibiotic output $(4,5,13)$. When they occur, these changes are thought to be caused by different social cues that indicate the presence of competitors. These can include nutrient stress, such as iron depletion, or factors that cause cellular damage or predict immediate danger, like antibiotics or quorum-dependent regulators of antibiotic production, such as $\gamma$-butyrolactones $(3,14-16)$. However, as yet, we remain unable to predict the generality of these responses or the phenotypic or genomic factors that regulate them. Here, building on the framework of the competition sensing hypothesis, we set out to test if strains respond antagonistically to competitors that cause them harm. In addition, we investigate whether strains respond to competitive cues that we expect to be produced by strains with similar primary and secondary metabolisms due to shared resource requirements or mechanisms of antibiotic regulation. Because these traits are phylogenetically conserved $(6,17-20)$, this predicts that Streptomyces will be more likely to respond to social cues from closely related species.

To examine the social factors that regulate antibiotic production in streptomycetes, we studied antagonistic interactions between 24 different strains across a broad phylogenetic range in two nutrient environments. First, in each nutrient environment, we tested all possible pairwise interactions between these strains $(24 \times 24=576)$ by growing them as colonies and then testing if they could inhibit the growth of each other strain by inoculating these on top of the focal colony (Fig. 1). Next, we tested if growth in coculture with a second strain altered the inhibitory behaviors we recorded during pairwise interactions. These three-way interactions consisted of $24 \times 24$ interactions, which we measured against 24 target strains to score 13,824 combinations in each nutrient environment. This allowed us to compare the inhibitory capacity of strains during solitary growth, reflecting constitutive expression, to their behavior after interacting with a competitor during coculture (Fig. 1). These approaches allowed us to directly test if altered antibiotic production during growth in coculture could be predicted as a function of the phenotype or genotype of the competitor. 


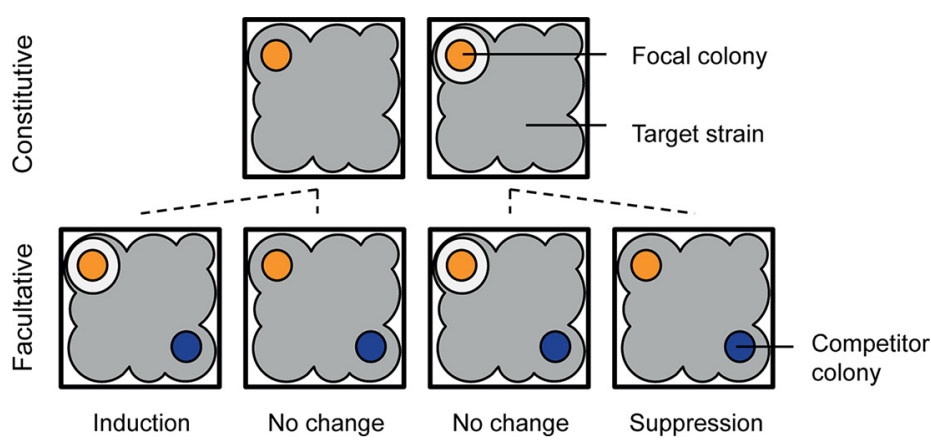

FIG 1 Schematic of constitutive and facultative inhibition assays. Focal strains (orange) were tested for their capacity to inhibit each target strain (gray) inoculated on top of the focal colony in a soft agar overlay. Inhibition was detected as a zone of clearance surrounding the colony. All 24 strains were tested as both focal and target strains, leading to 576 possible assays for constitutive antibiotic production. For the facultative assays, a second colony was inoculated $1 \mathrm{~cm}$ away, designated the competitor, that could interact with the focal strain through diffusible molecules. All 24 strains were tested each as the focal, competitor, and target strain, resulting in $24 \times 24 \times 24=13,824$ assays. All assays were conducted under both high- and low-resource conditions. Comparison of the ability of the focal strain to inhibit the target in the constitutive and facultative assays revealed whether antibiotic production was induced, suppressed, or unchanged.

\section{RESULTS}

Constitutive antagonism. We first measured constitutive antibiotic production by growing each strain on a defined minimal medium (MM) and then testing if it could inhibit an overlay of each target strain (Fig. 1). These results formed the baseline against which we examined facultative responses. Similar to antagonistic interactions in Streptomyces and other microbes $(4,6,21)$, these assays revealed that approximately half of all possible pairwise interactions were inhibitory (47.7\%) (Fig. 2A, top left triangles). We used multilocus sequence typing (MLST) to infer the phylogeny of the strains. Next, we identified the biosynthetic gene clusters (BGCs) in the complete genomes of these strains by using the bioinformatics tool antiSMASH (22). This revealed considerable variability in the number of secondary metabolite BGCs within each genome (mean \pm standard error $[S E]=34 \pm 1.85$; range $=22$ to 64 ) (see Fig. S2 in the supplemental material).

The antagonistic behavior of each strain against the 24 possible targets generated a unique inhibition fingerprint, which we designate the inhibition phenotype. We calculated the dissimilarity in inhibition phenotypes to quantify whether strains inhibit the same or different targets and found a significant correlation between inhibition phenotype dissimilarity and phylogenetic distance (Fig. 2B) (Mantel test, $P<0.001, r=0.27$ ). This result indicates that closely related strains inhibit the same targets. We then tested if this was due to the possibility that related strains produce similar antagonistic compounds. To address this, we grouped all BGCs identified in the genomes into gene cluster families by using BiG-SCAPE (23) and calculated Jaccard distances between the strains based on their shared BGCs. This analysis revealed that BGC distance is significantly correlated with inhibition phenotype (Mantel test, $P<0.001, r=0.43$ ) (Fig. 2C), supporting the idea that related strains produce similar secondary metabolites.

Consistent with the idea that closely related strains are more likely competitors, strains showed a stronger tendency to inhibit closely related targets (logistic regression, $P<0.001$, McFadden $R^{2}=0.02, n=536$ ). As BGCs often also provide resistance against the product they encode, we expected that strains with a high degree of BGC similarity would not inhibit each other. Indeed, strains were most likely to inhibit targets that are closely related but have dissimilar BGCs (logistic regression, $P_{\text {phylogenetic distance }}<0.001$, $P_{\text {BGC distance }}=0.046$, McFadden $R^{2}=0.02, n=536$ ) (Fig. 2D). In contrast to another study that examined inhibitory interactions between phylogenetically diverse bacteria (21), we found no association between the probability of inhibition and the metabolic overlap 

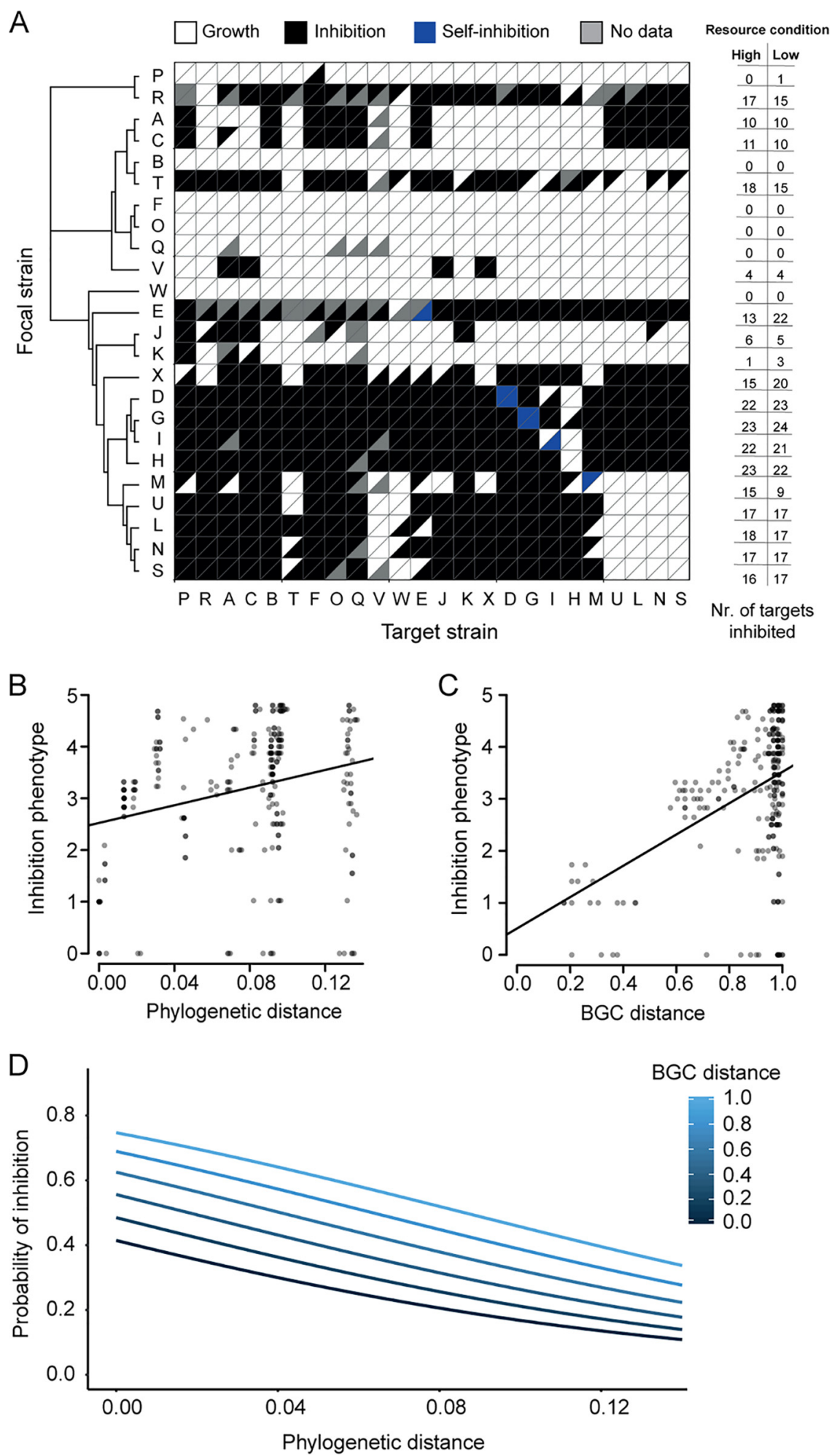

FIG 2 Constitutive antagonism. (A) Inhibition matrix sorted by multilocus sequence typing (MLST) relatedness. Triangles indicate whether a target strain showed growth (white) or was inhibited (black) by the focal strain. Each square is divided into two triangles: the upper triangle shows the results under high-resource conditions, while the lower triangle shows the results under low-resource conditions. Selfinhibition is denoted in blue. Missing data due to inconsistent results are shown in gray. Panels B to D show results of assays conducted at high resource levels. (B) Correlation between inhibition phenotype dissimilarity (Euclidian distance determined by calculating the dissimilarity between focal strain inhibition phenotypes) and phylogenetic distance (Mantel test, $P<0.001, r=0.27, n=552$ ) or (C) biosynthetic gene

(Continued on next page) 
between strains, assessed as the ability to grow on 95 different carbon sources by using Biolog plates.

Altered inhibition during coculture. Our results show that streptomycetes constitutively produce antibiotics that inhibit closely related strains. However, constitutive antibiotic production does not account for facultative changes that are caused by cues from other strains. We measured facultative responses by inoculating each strain next to a competitor and then assessing if it could inhibit the growth of the different target strains, as described above. This allowed us to directly compare the inhibitory capacity of each focal strain in the presence and absence of each competitor (Fig. 1). A focalcompetitor interaction was scored as "induced" if the focal strain was able to inhibit any of the target strains that it was unable to inhibit when it was grown on its own and "suppressed" if the opposite occurred. By this approach, a focal strain could be both induced and suppressed by the same competitor against different target strains. The results of these assays, shown in Fig. 3A, confirm that facultative responses are extremely widespread. The inhibition phenotype was changed by a competitor in approximately half of the focal-competitor interactions (48\%), meaning that the focal strain was induced or suppressed against at least one target strain in the presence of a given competitor. These changes dramatically altered the inhibition phenotype of the focal strain and changed the total number of strains that each focal strain could inhibit (Fig. 3B). Strains also changed their inhibition phenotype in response to a competitor colony of the same strain (58\% [diagonal in Fig. 3A]). Overall, we observed induction in $33 \%$ of all tested focal-competitor interactions and suppression in $45 \%$. There was considerable variability in the responsiveness of strains to competitors; whereas some strains responded to none of the competitors, others responded to nearly all of them (induced, 0 to 20; suppressed, 0 to 22) (Fig. 3C). On average each focal strain was induced by $7.4 \pm$ 1.5 (mean \pm SE) competitors and suppressed by $9.6 \pm 1.7$. In many cases, a given strain was both induced and suppressed by the same competitor against different targets (Fig. 3A). Although this led to a distinct inhibition phenotype, compared to the focal strain grown alone, it may not have changed the total number of inhibited targets.

Competition sensing predicts that bacteria will change their behavior in response to antagonistic competitors that they detect by sensing cell damage (3). We define a competitor as antagonistic if it inhibits the focal strain during the constitutive assay. Although we found that induction of the focal strain was significantly related to whether or not its competitor was antagonistic (logistic regression, $P<0.001$, McFadden $R^{2}=$ $0.06, n=354$ ), the direction of this result did not match our expectations (Fig. 4A, black bars). Unexpectedly, antibiotic production in the focal strain was nearly twice as likely to be induced by a nonantagonistic competitor (probability of induction of 0.41 versus 0.22). This indicates that cell damage was not a strong cue for antibiotic induction. Other ways that focal cells could sense competitors is if they detect compounds they produce, such as antibiotics and quorum sensing signals, or by experiencing nutrient stress due to resource competition. Since both primary metabolism and secondary metabolism are correlated with phylogenetic distance, we examined if induction was correlated with phylogenetic distance. As predicted, focal strains are more frequently induced by a closely related competitor (logistic regression, $P<0.001$, McFadden $R^{2}=0.02, n=487$ ) (Fig. 4B, solid line). To examine if this effect was associated with the production of similar secondary metabolites, we tested if differences in induction could be explained by BGC similarity. Indeed, focal strains are more likely induced by competitors with which they share more BGCs (logistic regression, $P<0.001$, McFadden $R^{2}=0.04, n=487$ ) (Fig. 4C, solid line). This suggests that cues for induction rely more on the detection of secreted compounds, rather than the damage these compounds cause. This makes cases of self-

FIG 2 Legend (Continued)

cluster (BGC) distance (Mantel test, $P<0.001, r=0.43, n=552$ ). Dots are semitransparent: darker areas represent overlapping data points. (D) Logistic regression between the probability of inhibition and phylogenetic and biosynthetic gene cluster (BGC) distance $\left(P_{\text {phylogenetic distance }}<0.001, P_{\mathrm{BGC} \text { distance }}=0.046\right.$, McFadden $R^{2}=0.02, n=536$ ). 
High resource conditions

A

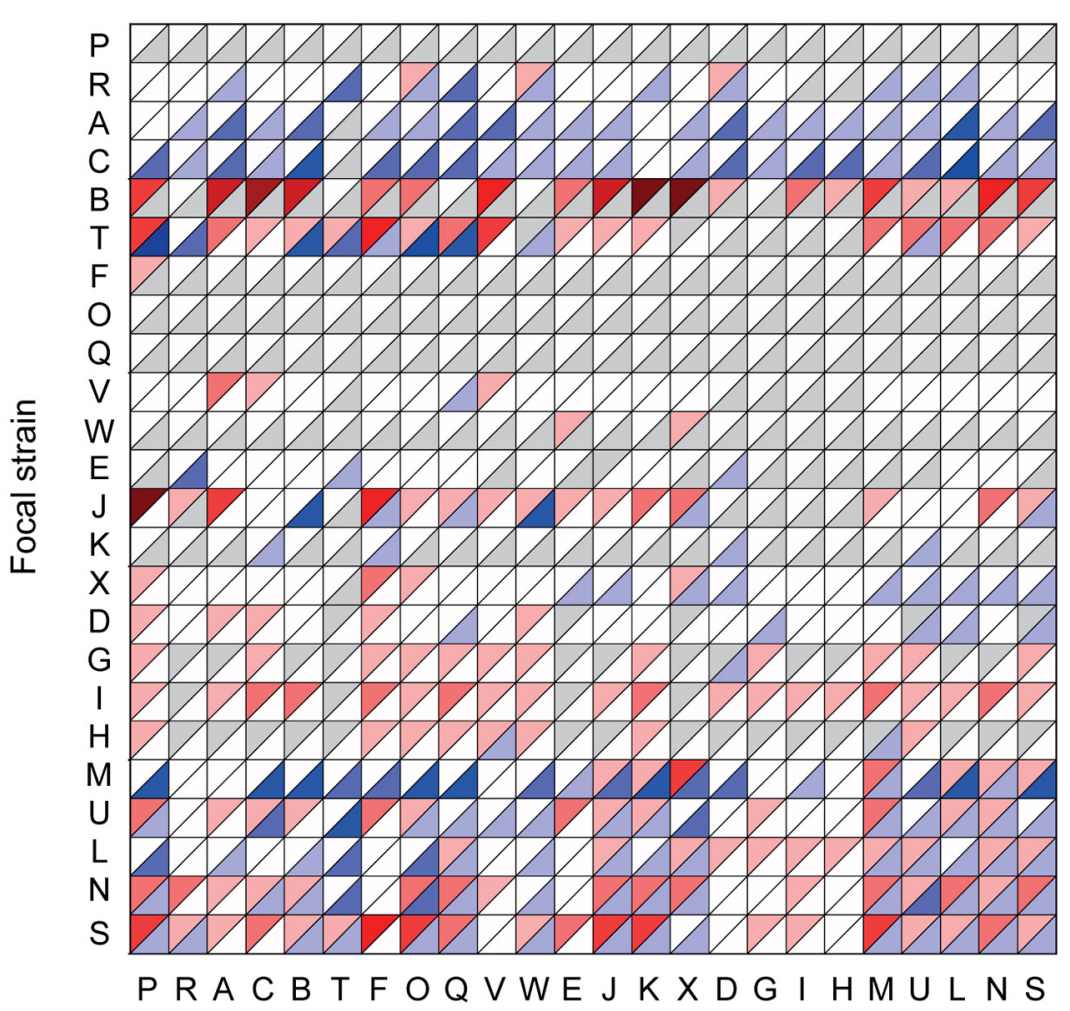

Competitor strain
B

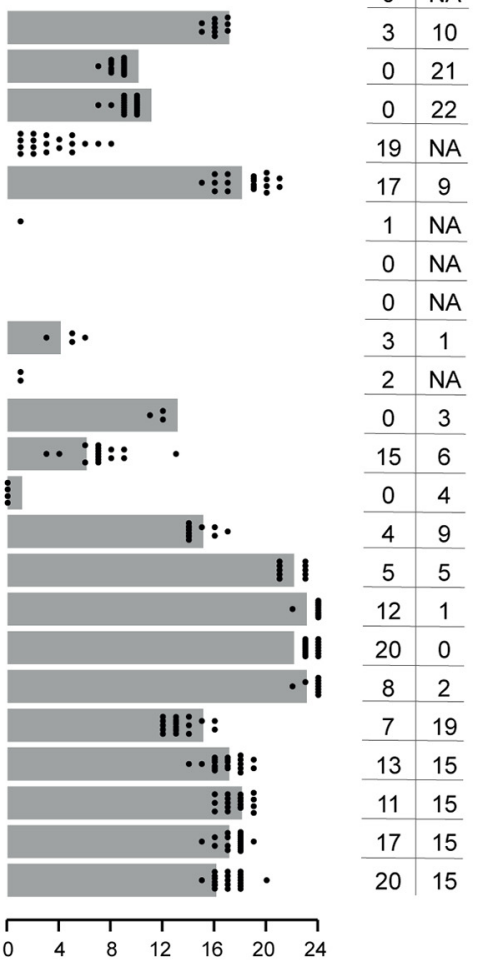

Total number of targets inhibited

FIG 3 Altered antagonism during coculture under high-resource conditions. (A) Interaction heat map showing changes to target strain inhibition when a focal strain is grown in coculture with a competitor. Each square is divided into two triangles: the upper triangle shows induction in red, while the lower triangle shows suppression in blue. Gray triangles indicate that either induction or suppression was not possible for this focal strain, due to the result in the constitutive assay. (A strain that does not inhibit any targets cannot be suppressed, while a strain that inhibits all targets cannot be induced.) (B) Gray bars indicate the number of target strains inhibited by the focal strain when grown alone. Black dots indicate the net number of target strains inhibited by the same focal strain if it was induced and/or suppressed during coculture with one of the 24 possible competitors. Dots showing the same number of inhibited target strains as the gray bar indicate that a competitor strain causes an equal level of induction and suppression against targets, resulting in no net change. (C) Number of competitors that induce or suppress each focal strain. Cases where suppression is not possible due to the absence of constitutive inhibition are denoted as "NA."

induction, where the focal and competitor colonies are of the same strain, also likely due to a local increase in the concentration of autoinducers, such as $\gamma$-butyrolactones.

In addition to induction, we found that antibiotic production was also commonly suppressed in the presence of competitors. Although this strategy can be perceived as benefiting the competitor strain if it prevents a focal strain from producing a potentially harmful antibiotic, it could also benefit the suppressed strain by allowing it to redirect energy toward other functions. However, we found no relationship between suppression and the competitor's ability to inhibit the focal strain (logistic regression, $P=0.83$, McFadden $\left.R^{2}=0.025\right)$. Suppression was also not associated with phylogenetic or BGC distance (logistic regression, $P=0.94$, McFadden $R^{2}<0.001, n=366$, and $P=$ 0.202 , McFadden $R^{2}=0.003, n=366$, respectively).

Effect of resource stress on inhibition. To address the role of nutrient limitation in antibiotic production, we tested whether constitutive or facultative inhibition changed if the carbon source concentration was reduced by 10-fold (Fig. 2A, bottom right 

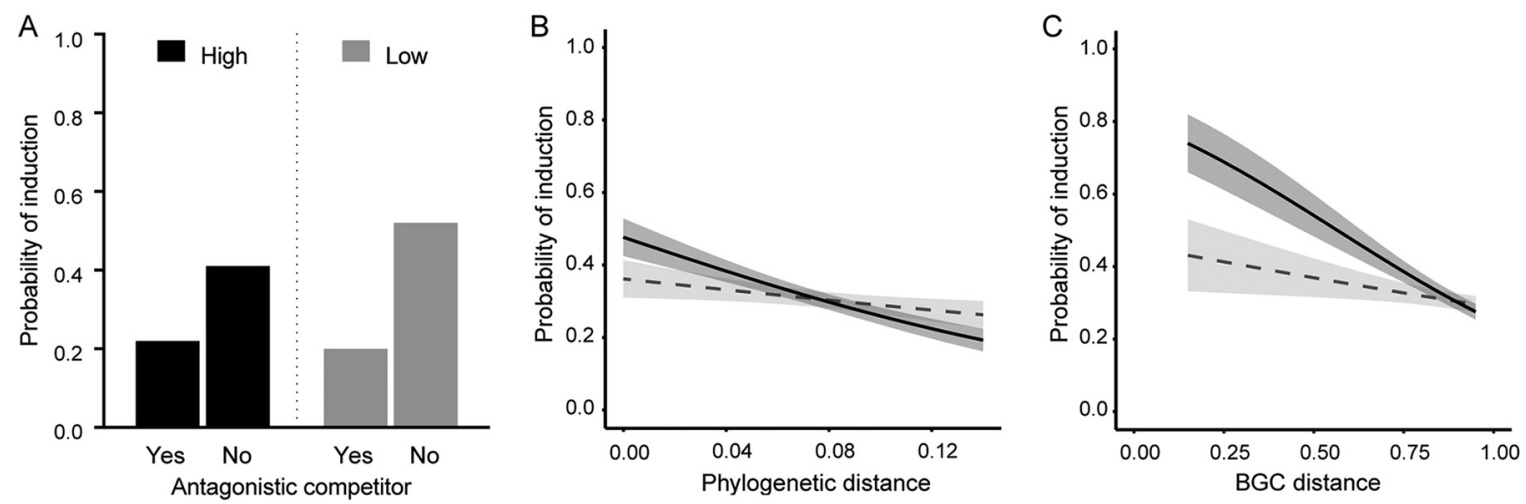

FIG 4 Induction during coculture. (A) The probability that a focal strain is induced is lower when the competitor is antagonistic to the focal strain under both high-resource (logistic regression, $P<0.001$, McFadden $R^{2}=0.06, n=354$ ) and low-resource (logistic regression, $P<0.001$, McFadden $\left.R^{2}=0.12, n=419\right)$ conditions. (B) Logistic regressions between the probability of induction and phylogenetic distance under high-resource (black line) and low-resource (dashed line) conditions $\left(P<0.001\right.$, McFadden $R^{2}=0.02$, $n=487$ and $P=0.205$, McFadden $R^{2}=0.00292, n=445$, respectively) or (C) logistic regressions between the probability of induction and BGC distance under high-resource (black line) and low-resource (dashed line) conditions $\left(P<0.001\right.$, McFadden $R^{2}=0.04, n=487$ and $P=0.174$, McFadden $R^{2}=0.003, n=445$, respectively). Shaded areas indicate SE.

triangles). The frequency of constitutive inhibition was marginally higher under these conditions: $49.2 \%$ versus $47.7 \%$ of all pairwise interactions were inhibitory on low- versus high-resource medium, and only $6.7 \%$ of pairwise interactions differed between the two resource conditions (McNemar's $\chi^{2}=0, \mathrm{df}=1, P=1$ ). Likewise, we found a strong correlation between the inhibition phenotypes of the strains at both resource concentrations (Mantel test, $r=0.93, P<0.001$ ), with phylogenetic and BGC distances both significantly correlated with inhibition phenotype and in the same direction as in the high-resource concentration (Mantel test, $P<0.001, r=0.30$, and $P<0.001$, $r=0.39$, respectively) (see Fig. S1 in the supplemental material). As at the higher glycerol concentration, strains are more likely to inhibit closely related targets with dissimilar BGCs (logistic regression, $P_{\text {phylogenetic distance }}<0.001, P_{\mathrm{BGC}}$ distance $=0.019$, McFadden $R^{2}=0.02, n=526$ ) (Fig. S1).

Streptomyces focal strains responded differently to the presence of a competitor under different resource conditions (Fig. 5) (McNemar's $\chi^{2}=5.43, \mathrm{df}=1, P<0.05$ ), with a change in inhibition phenotype in $56.1 \%$ versus $49.1 \%$ under low- versus highresource conditions, respectively (Fig. 6). While we expected more induction due to resource stress, in accordance with the competition sensing hypothesis, the incidence of induction was slightly lower at lower resource levels (30.2\% versus $33.0 \%$ ). In contrast we observed a dramatic increase in suppression, from $45.0 \%$ to $59.1 \%$ (Fig. 6). Just as at the higher resource level, strains were more likely to be induced by competitors that did not inhibit them (Fig. 4A, gray bars) (logistic regression, $P<0.001$, McFadden $\left.R^{2}=0.12, n=419\right)$. In contrast to the higher-resource conditions, neither phylogenetic nor BGC similarity was associated with induction in the low-resource environment (Fig. 4B and $C$, dashed lines) (logistic regression, $P=0.205$, McFadden $R^{2}=0.00292$, $n=445$ and $P=0.174$, McFadden $R^{2}=0.003, n=445$, respectively). Suppression was still not associated with any of the factors that we tested, suggesting that antibiotic suppression may be a general reaction to resource stress in streptomycetes.

\section{DISCUSSION}

Competitive and social interactions between neighboring microbial cells in soil are common as different species vie for space and resources. One of the ways that bacteria compete is by secreting toxins like antibiotics or bacteriocins, but the production of these compounds is typically studied without consideration of this biotic context. Both theory and experiments have shown that this perspective is limited because it neglects 
Low resource conditions

A

\begin{tabular}{lllll}
\hline-7 & Suppressed & 0 & Induced & 7 \\
\hline
\end{tabular}

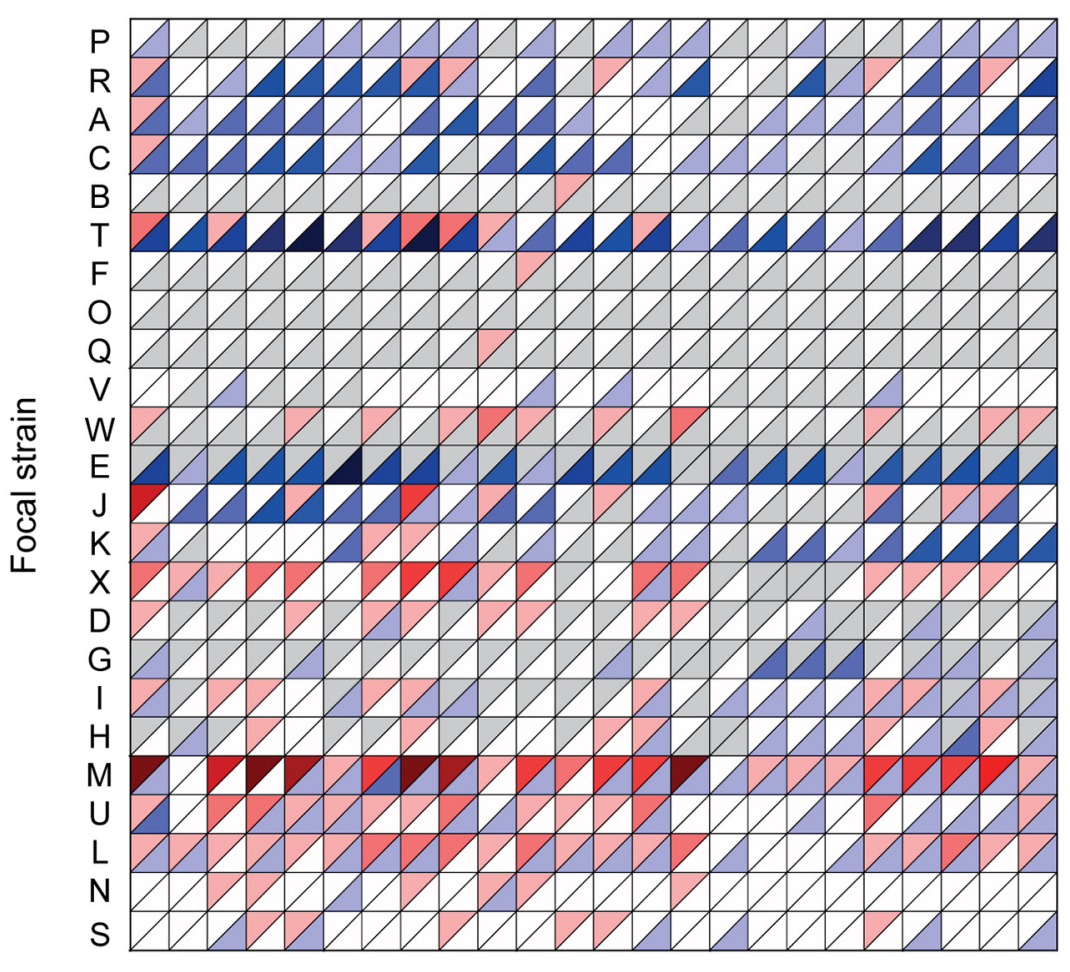

PRACBTFOQVWE JKXDG I HM ULN S
B

I
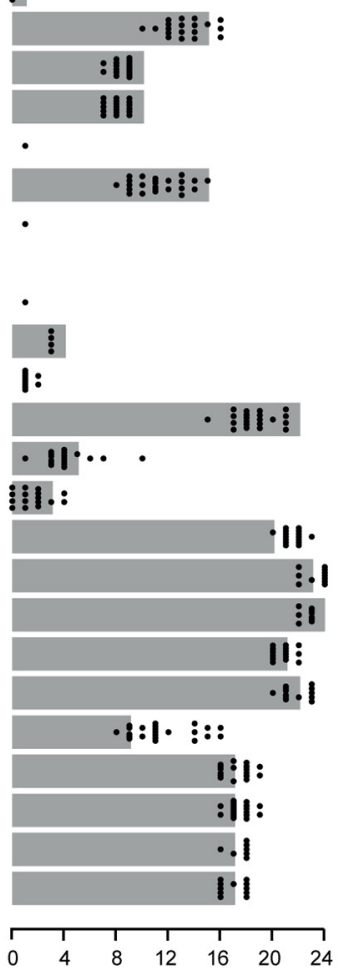

Total number of targets inhibited

C

\begin{tabular}{c|c} 
Ind & Supp \\
\hline 0 & 15 \\
\hline 6 & 16 \\
\hline 1 & 19 \\
\hline 1 & 20 \\
\hline 1 & NA \\
\hline 7 & 24 \\
\hline 1 & NA \\
\hline 0 & NA \\
\hline 1 & NA \\
\hline 0 & 4 \\
\hline 11 & NA \\
\hline NA & 23 \\
\hline 8 & 16 \\
\hline 3 & 14 \\
\hline 16 & 3 \\
\hline 8 & 4 \\
\hline NA & 9 \\
\hline 9 & 14 \\
\hline 6 & 8 \\
\hline 22 & 19 \\
\hline 14 & 12 \\
\hline 20 & 16 \\
\hline 6 & 2 \\
\hline 16 & 6
\end{tabular}

Competitor strain

FIG 5 Altered antagonism during coculture under low-resource conditions. (A) Interaction heat map showing change in target strain inhibition when a focal strain is grown in coculture with a competitor. Each square is divided into two triangles: the upper triangle shows induction in red, while the lower triangle shows suppression in blue. Gray triangles indicate that either induction or suppression was not possible for this focal strain, due to the result in the constitutive assay. (A strain that does not inhibit any targets cannot be suppressed while a strain that inhibits all targets cannot be induced.) (B) Gray bars indicate the number of strains inhibited by the focal strain when grown alone. Black dots indicate the net number of target strains inhibited by the same focal strain if it was induced and/or suppressed during coculture with one of the 24 possible competitors. Dots showing the same number of inhibited target strains as the gray bar indicate that a competitor strain causes an equal level of induction and suppression against targets, resulting in no net change. (C) Number of competitors that induce or suppress each focal strain. Cases where suppression is not possible due to the absence of constitutive inhibition are denoted as "NA."

factors that induce or suppress toxins and fails to identify toxins whose production is dependent on competitive interactions $(4,5,24-27)$. In this context, the aims of our work were two-fold: first to establish to what extent the role of social interactions affect antibiotic production in common soil microbes of the Streptomycetaceae, and second to identify factors that are predictive of competition-mediated responses.

By comparing levels of antibiotic production in the absence and presence of another species in coculture, termed constitutive production and facultative production, respectively, we found that production was induced in $\sim 1 / 3$ of cocultures, under both high- and low-resource conditions. These results considerably expand on results from earlier studies that describe changes in secondary metabolite production and inhibition in a smaller set of actinomycetes or against a smaller set of target species (4-6). The latter aspect is especially important because susceptibility varies markedly between strains; studies with fewer targets may therefore underestimate the frequency of facultative changes to antibiotic production. Induction was strongly predicted by 


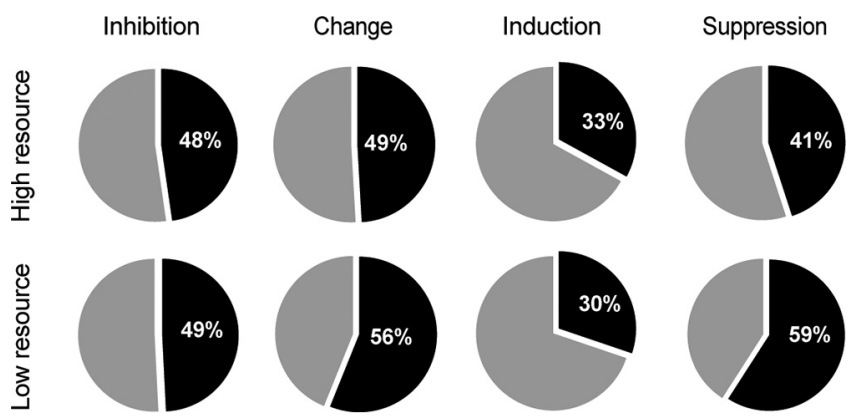

FIG 6 Constitutive and facultative inhibition under high- and low-resource conditions. Shown are comparisons of the total amount of inhibition, change in inhibition due to competition, and induction and suppression found under low- and high-resource conditions.

phylogenetic distance in our study, with closely related strains more likely to inhibit each other. Previous studies also identified a correlation between phylogenetic distance and inhibition in Streptomyces $(6,13)$. However, one of these pointed in the opposite direction, showing closely related strains as less likely to inhibit each other, which could possibly be due to the differences in the method of determining growth inhibition (13). The weak significant relationships identified in the present study suggest that other untested factors, beside the parameters tested here, may be important. An earlier study linked the association between induction and phylogenetic distance to nutrient use, as closely related strains compete for the same resources, but did not test for overlap in secondary metabolites (6). The same study found that streptomycetes increase antibiotic production when grown with susceptible competitors, without assessing the impact of whether or not the competitor could reciprocally inhibit the focal strain. Unexpectedly, we found that a focal strain was less likely to be induced during coculture if it was grown with an inhibitory competitor. In other words, while coculture frequently altered antibiotic production, this was not evidently driven by cellular damage caused by the second strain, as specifically predicted by the competition sensing hypothesis (3). Instead, our results suggest that cells are more likely to induce antibiotic production in response to cues that are correlated with phylogenetic distance, rather than direct harm itself. For example, under high-resource conditions strains that share BGCs are more likely to induce each other. This suggests two possible sources for cues. First, antibiotic intermediates or antibiotics themselves, can serve as inducers of antibiotic production or resistance (16). These responses can prevent autotoxicity or killing by neighboring clonemates and also act as regulators of the expression of their own biosynthetic gene cluster $(26,28)$. Because resistance is often encoded in the antibiotic biosynthetic gene cluster, self-inhibition, which was observed in few instances, could be due to not-yet-expressed resistance in spores during challenge with a high dose of antibiotic, or alternatively by the production of germination inhibitors, such as germicidin, that inhibit the germination of conspecific spores (29). Second, related strains that share one or more BGCs may be more likely to utilize the same, or similar, secreted factors that induce antibiotic production: e.g., the quorumdependent $\gamma$-butyrolactone signals. Streptomyces cells contain multiple receptors for cognate and noncognate $\gamma$-butyrolactones, thereby allowing them to detect these signals as a precursor of the antibiotics another strain might produce $(15,30,31)$. Similar eavesdropping of quorum-dependent signals has been observed for bacteriocins in Streptococcus pneumoniae, which leads to cross-induction of strain-specific antimicrobials (7). Testing this idea in Streptomyces using chemically synthesized signals and reporter strains remains an important objective for future work.

When we repeated our assays at 1/10 the glycerol concentration, constitutive expression was only marginally changed; however, these lower resource concentrations led to slightly reduced induction rates and a marked increase in suppression. 
Moreover, the associations between induction and phylogenetic distance and BGC distance disappeared. These results indicate that antibiotic regulation integrates information about the competitive environment as well as environmental resource availability, leading strains to respond differently when exposed to a combination of competitive cues and resource stress than when exposed to only one of these. Links between nutrient sensing or carbon catabolite repression and antibiotic production in streptomycetes are well established. For example, under nutrient-rich conditions $\mathrm{N}$-acetylglucosamine blocks morphogenesis and antibiotic production, while it has the opposite effect under nutrient-poor conditions $(32,33)$. A second possibility may be that competition exacerbates nutrient stress overall, leading to a general suppressive response that does not depend on the particulars of the competitor. By this view, suppression is best considered a generic response to nutrient stress, rather than the result of a specific action by the second strain. Other possibilities exist, such as degradation of exogenous enzymes or coercion or manipulation by the competitor colony. These strategies could be amplified by lowered nutrients to reflect a switch from offense to defense. This result indicates that further work will need to consider responses other than antibiotic production when examining the behavior of cells in coculture. For example, strains may respond to nutrient stress from competitors by redirecting energy used for antagonism toward functions that help them to avoid competition: e.g., hyphal growth in the direction opposite the competing strain or increased sporulation. Whereas the first possibility would contribute to an escape in space, the latter would allow an escape in time, leaving spores to germinate when nutrient stress is relieved. These alternative responses, which are equivalent to a "fight or flight" decision, might be anticipated if there are trade-offs between antibiotic production and other aspects of development, as we have found in Streptomyces coelicolor (34).

In summary, our results provide strong evidence that antibiotic production by streptomycetes is highly responsive to their social and resource environment. We establish the importance of BGC similarity on antibiotic induction, suggesting a role for shared regulatory compounds, and show that suppression and possibly escape, as a means of "flight," should be more thoroughly examined as a response to interference competition. This is equally important for many of the other mechanisms that bacteria use to regulate inter- and intraspecific warfare (35). It will also be crucial to examine these responses in experiments that more closely approximate the natural environment, including environments with increased spatial heterogeneity and decreased diffusion, and where local interactions are maintained over longer periods of time. Similarly, an important next step is to determine how these social interactions influence competitive outcomes, as has been done for constitutive antibiotic production between competing species $(1,2,36)$. Together, these approaches will lead to a fuller understanding of the role of antibiotic production in natural soils and the factors that maintain microbial diversity. In addition, they will help to identify factors that can be used to induce cryptic antibiotic BGCs in Streptomyces as potential drug leads.

\section{MATERIALS AND METHODS}

Strains and culturing conditions. The panel of 24 Streptomycetaceae strains used in this study (see Table S1 in the supplemental material) included 21 strains isolated from a single soil sample from the Himalayan Mountains collected at 5,000 m near a hot water spring (37). These 21 strains were selected due to their consistent phenotypes and the ability to sporulate under our lab growth conditions. The remaining three strains were well-characterized lab strains: Streptomyces coelicolor A3(2) M145, Streptomyces griseus IFO13350, and Streptomyces venezuelae ATCC 10712.

High-density spore stocks were generated by culturing on soy flour-mannitol (SFM) agar ( $20 \mathrm{~g}$ soy flour, $20 \mathrm{~g}$ mannitol, $20 \mathrm{~g}$ agar per liter) or on R5 agar $\left\{103 \mathrm{~g}\right.$ sucrose, $0.42 \mathrm{~g} \mathrm{~K}_{2} \mathrm{SO}_{4}, 10.1 \mathrm{~g} \mathrm{MgCl}_{2}, 50 \mathrm{~g}$ glucose, $0.1 \mathrm{~g}$ Casamino Acids, $5 \mathrm{~g}$ yeast extract, $5.7 \mathrm{~g}$ TES [ $\mathrm{N}$-tris(hydroxymethyl)methyl-2-aminoethanesulfonic acid], $2 \mathrm{ml}$ R5 trace element solution, and $22 \mathrm{~g}$ agar per liter\}. After 3 to 4 days of growth, spores were harvested with a cotton disc soaked in $3 \mathrm{ml} 20 \%$ glycerol, and spores were extracted from the cotton by passing the liquid through an 18-gauge syringe to remove the vegetative mycelium. Titers of the resulting spore stocks were determined and stored at $-20^{\circ} \mathrm{C}$.

Multiwell master plates were prepared by diluting the high-density spore stocks to $1 \times 10^{6}$ spores $\mathrm{ml}^{-1}$ in deionized water, and these plates were stored at $-20^{\circ} \mathrm{C}$. The glycerol concentration after the dilution of stocks was always lower than the concentration of glycerol added as a carbon source to the medium. 
To perform the interaction assays, approximately $1 \mu \mathrm{l}$ of the focal strain (and when indicated $1 \mu \mathrm{l}$ of the competitor strain) was replicated on a 25 -grid plate (Thermo Fisher Scientific, Newport, United Kingdom) using a custom-built multipin replicator (EnzyScreen BV, Heemstede, The Netherlands) from a frozen master plate. Each well of the 25 -grid plate contained $2 \mathrm{ml}$ minimal medium (MM) $(500 \mathrm{mg}$ L-asparagine [Duchefa Biochemie, The Netherlands], $500 \mathrm{mg} \mathrm{KH}_{2} \mathrm{PO}_{4}$ [Duchefa Biochemie], $200 \mathrm{mg} \mathrm{MgSO} \cdot \cdot 7 \mathrm{H}_{2} \mathrm{O}$ [Duchefa Biochemie], $10 \mathrm{mg} \mathrm{Fe} \mathrm{SO}_{4} \cdot 7 \mathrm{H}_{2} \mathrm{O}$ [Sigma-Aldrich, St. Louis, MO], and $20 \mathrm{~g}$ agar [TM Duché \& Sons Ltd, Wilmslow, Cheshire, UK] per liter [pH 7.2] supplemented with either 0.05 or $0.5 \%$ glycerol). After 4 days of growth at $30^{\circ} \mathrm{C}$, a $1-\mathrm{ml}$ overlay $(0.8 \%$ agar $\mathrm{MM})$ containing $1.6 \times 10^{5}$ spores $/ \mathrm{ml}$ was added on top. After 24 to $48 \mathrm{~h}$ of incubation at $30^{\circ} \mathrm{C}$ (depending on the growth speed of the target strain), $1 \mathrm{ml}$ of the dye resazurin (Cayman Chemical Company, Ann Arbor, Ml) was added to each well at a concentration of $50 \mathrm{mg}$ lit$\mathrm{er}^{-1}$, and then the mixture was incubated for half an hour before the surplus was removed. Change in color of this redox dye from blue to pink was used as a measure of growth of the target strain, as resazurin (blue) is changed to resorufin (pink) by metabolically active cells. Pictures were taken of every plate, and these were scored for the presence or absence of inhibition zones around the colony/colonies. Every interaction was assessed at least in duplicate. When the results of assays were inconsistent, the particular interaction was repeated. In some cases, it was impossible to score assay results due to spreading of the spores from a colony into the overlay agar with the target strain. This resulted in an easily recognizable "swirl" of growth coming from a colony, and as such, we excluded these results (designated "missing data").

Whole-genome sequencing. Whole-genome sequencing was performed for all strains for which a full genome sequence was not yet available to perform genome mining and to generate a phylogenetic tree. As described before (38), strains were grown in liquid culture containing 50\% YEME-50\% TSBS with $5 \mathrm{mM} \mathrm{MgCl}$ and $0.5 \%$ glycine at $30^{\circ} \mathrm{C}$ and with centrifugation at $250 \mathrm{rpm}$ for 2 days. After centrifugation, the pellet was resuspended in TEG buffer with $1.5 \mathrm{mg} \mathrm{ml}^{-1}$ lysozyme, and after $1 \mathrm{~h}$ of incubation at $30^{\circ} \mathrm{C}$, the reaction was stopped by adding 0.5 volume of $2 \mathrm{M} \mathrm{NaCl}$. DNA was extracted using a standard phenol-chloroform extraction, followed by DNA precipitation and washing in isopropanol and $96 \%$ ethanol. Dried DNA was resuspended in Milli-Q water and then treated with $50 \mu \mathrm{g} \mathrm{ml}^{-1}$ of RNase and incubated at $37^{\circ} \mathrm{C}$ for $1 \mathrm{~h}$. Following RNase treatment, the mixture was purified and cleaned as described above, after which the purified DNA was washed with $70 \%$ ethanol and resuspended in Milli-Q water. Paired-end sequence reads were generated using the Illumina HiSeq2500 system at BaseClear. De novo assembly was performed using the "De novo assembly" option of the CLC Genomics Workbench version 9.5.1, and the genome was annotated using the BaseClear annotation pipeline based on the Prokka Prokaryotic Genome Annotation System (version 1.6).

Using the complete genomes, multilocus sequence typing (MLST) was performed as described by Guo et al. (39). For this purpose, we used the sequences of six housekeeping genes ( $a t p D$, gyrB, recA, $r p o B, \operatorname{trp} B$, and $16 \mathrm{~S} r \mathrm{rNA}$ ) that were shown to give good resolution for the $S$. griseus clade. For the already available sequenced genomes, the sequences for $S$. coelicolor (strain $V$ ) were downloaded from StrepDB (http://strepdb.streptomyces.org.uk) and used to BLAST search against the genome sequences of S. venezuelae ATCC 10712 (txid 54571) (strain W), S. griseus subsp. griseus NBRC 13350 (txid 455632), and MBT66 (strain P) in the NCBI database. For all sequenced genomes, the genes of interest were located from the annotated genome or were searched in a database constructed with the genomes in Geneious (Geneious 9.1.4). Each gene was aligned and trimmed before the six sequences for each strain were concatenated in frame and used to construct a neighbor-joining tree using Geneious to reveal the phylogenetic distances between the strains.

Analysis of biosynthetic gene clusters. Biosynthetic gene clusters were identified within each genome with antiSMASH version 4.0 run with default parameters (22). BiG-SCAPE was used to calculate the pairwise distances between all BGCs, using default parameters and a cutoff of 0.5 as a threshold for similarity (23). This generated a BGC presence/absence matrix that we used to calculate a Jaccard distance between each pair of genomes to define the BGC distance between the strains.

Resource use. Carbon source utilization of each strain was tested using Biolog SFP2 plates (Biolog, Hayward, CA), on which growth on 95 carbon sources can be assessed. Plates were inoculated as described by Schlatter et al. (40). Briefly, strains were grown on MM with $0.5 \%$ glycerol for 7 days before spores were swabbed into a $0.2 \%$ carrageenan solution and adjusted to an optical density at $590 \mathrm{~nm}$ $\left(\mathrm{OD}_{590}\right)$ of 0.2 to 0.24 . This solution was diluted 10 times in $0.2 \%$ carrageenan, and $100 \mu$ l of this dilution was added to each well. Plates were incubated at $30^{\circ} \mathrm{C}$ for 3 days before the absorbance of each well at $590 \mathrm{~nm}$ was measured using a Spark 10M plate reader (Tecan, Switzerland). All strains were assessed in triplicate. For the analysis, the absorbance of the water control was subtracted for each well and the mean was taken. If the sample mean was not significantly different from 0 (one-sample $t$ test, $\alpha=0.05$ ), the value was adjusted to 0 . The Pearson correlation coefficient was calculated between all possible pairwise combinations of the strains, and the metabolic distance was calculated as 1 - correlation coefficient. Strain P showed extremely poor growth on the Biolog plates and was therefore excluded.

Statistics. All statistics were performed in R. Correlation between phylogenetic distance, metabolic dissimilarity, secondary metabolite distance, and inhibition and resistance phenotype was determined using Mantel tests. To establish whether antagonism and inhibition and induction and suppression are dependent, logistic regressions were performed. Logistic regression was also used to test for association between inhibition, induction or suppression and phylogenetic distance, and metabolic distance or BGC distance. For the logistic regressions, we excluded all self-self interactions, as these confound the analyses by having zero distance between the strains or test for self-inhibition. 
Data availability. The data generated in this study are deposited in Dryad (https://doi.org/10.5061/ dryad.wpzgmsbkw) and the genome sequences are deposited in GenBank under the corresponding MBT names.

\section{SUPPLEMENTAL MATERIAL}

Supplemental material is available online only.

FIG S1, PDF file, $0.5 \mathrm{MB}$.

FIG S2, PDF file, $0.2 \mathrm{MB}$.

FIG S3, PDF file, $0.3 \mathrm{MB}$.

FIG S4, PDF file, $0.3 \mathrm{MB}$.

TABLE S1, PDF file, $0.5 \mathrm{MB}$.

\section{ACKNOWLEDGMENTS}

This work was financially supported by grants 824.02 .003 and 731.014 .206 from the

Dutch National Science Foundation (NWO) to D.E.R. and to G.P.v.W., respectively.

The authors contributed in the following ways. S.W. and D.E.R. designed the experiments. S.W. performed the experiments and analyzed the data. A.K. and S.F.A.v.H. assisted with the (bio)informatics. S.W., D.E.R., and G.P.v.W. wrote the manuscript.

We declare there is no conflict of interest.

\section{REFERENCES}

1. Chao L, Levin BR. 1981. Structured habitats and the evolution of anticompetitor toxins in bacteria. Proc Natl Acad Sci U S A 78:6324-6328. https:// doi.org/10.1073/pnas.78.10.6324.

2. Westhoff S, Otto SB, Swinkels A, Bode B, van Wezel GP, Rozen DE. 2020. Spatial structure increases the benefits of antibiotic production in Streptomyces. Evolution 74:179-187. https://doi.org/10.1111/evo.13817.

3. Cornforth DM, Foster KR. 2013. Competition sensing: the social side of bacterial stress responses. Nat Rev Microbiol 11:285-293. https://doi.org/ 10.1038/nrmicro2977.

4. Abrudan MI, Smakman F, Grimbergen AJ, Westhoff S, Miller EL, van Wezel GP, Rozen DE. 2015. Socially mediated induction and suppression of antibiosis during bacterial coexistence. Proc Natl Acad Sci U S A 112: 11054-11059. https://doi.org/10.1073/pnas.1504076112.

5. Traxler MF, Watrous JD, Alexandrov T, Dorrestein PC, Kolter R. 2013. Interspecies interactions stimulate diversification of the Streptomyces coelicolor secreted metabolome. mBio 4:e00459-13. https://doi.org/10.1128/ mBio.00459-13.

6. Vaz Jauri P, Kinkel LL. 2014. Nutrient overlap, genetic relatedness and spatial origin influence interaction-mediated shifts in inhibitory phenotype among Streptomyces spp. FEMS Microbiol Ecol 90:264-275. https://doi .org/10.1111/1574-6941.12389.

7. Miller EL, Kjos M, Abrudan MI, Roberts IS, Veening JW, Rozen DE. 2018. Eavesdropping and crosstalk between secreted quorum sensing peptide signals that regulate bacteriocin production in Streptococcus pneumoniae. ISME J 12:2363-2375. https://doi.org/10.1038/s41396-018-0178-x.

8. Garbeva P, Silby MW, Raaijmakers JM, Levy SB, De Boer W. 2011. Transcriptional and antagonistic responses of Pseudomonas fluorescens Pf0-1 to phylogenetically different bacterial competitors. ISME J 5:973-985. https://doi.org/10.1038/ismej.2010.196.

9. Labeda DP, Goodfellow M, Brown R, Ward AC, Lanoot B, Vanncanneyt M, Swings J, Kim SB, Liu Z, Chun J, Tamura T, Oguchi A, Kikuchi T, Kikuchi H, Nishii T, Tsuji K, Yamaguchi Y, Tase A, Takahashi M, Sakane T, Suzuki KI, Hatano K. 2012. Phylogenetic study of the species within the family Streptomycetaceae. Antonie Van Leeuwenhoek 101:73-104. https://doi.org/10 .1007/s10482-011-9656-0.

10. Barka EA, Vatsa $P$, Sanchez L, Gaveau-Vaillant N, Jacquard C, MeierKolthoff JP, Klenk H-P, Clément C, Ouhdouch Y, van Wezel GP. 2016. Taxonomy, physiology, and natural products of Actinobacteria. Microbiol Mol Biol Rev 80:1-43. https://doi.org/10.1128/MMBR.00019-15.

11. Bibb MJ. 2005. Regulation of secondary metabolism in streptomycetes. Curr Opin Microbiol 8:208-215. https://doi.org/10.1016/j.mib.2005.02.016.

12. van der Heul HU, Bilyk BL, McDowall KJ, Seipke RF, van Wezel GP. 2018. Regulation of antibiotic production in Actinobacteria: new perspectives from the post-genomic era. Nat Prod Rep 35:575-604. https://doi.org/10 .1039/c8np00012c.

13. Vetsigian K, Jajoo R, Kishony R. 2011. Structure and evolution of
Streptomyces interaction networks in soil and in silico. PLoS Biol 9: e1001184. https://doi.org/10.1371/journal.pbio.1001184.

14. Traxler MF, Seyedsayamdost MR, Clardy J, Kolter R. 2012. Interspecies modulation of bacterial development through iron competition and siderophore piracy. Mol Microbiol 86:628-644. https://doi.org/10.1111/mmi .12008.

15. Zou Z, Du D, Zhang Y, Zhang J, Niu G, Tan H. 2014. A $\gamma$-butyrolactonesensing activator/repressor, JadR3, controls a regulatory mini-network for jadomycin biosynthesis. Mol Microbiol 94:490-505. https://doi.org/10 $.1111 / \mathrm{mmi} .12752$.

16. Wang W, Ji J, Li X, Wang J, Li S, Pan G, Fan K, Yang K. 2014. Angucyclines as signals modulate the behaviors of Streptomyces coelicolor. Proc Natl Acad Sci U S A 111:5688-5693. https://doi.org/10.1073/pnas.1324253111.

17. Kinkel LL, Schlatter DC, Xiao K, Baines AD. 2014. Sympatric inhibition and niche differentiation suggest alternative coevolutionary trajectories among Streptomycetes. ISME J 8:249-256. https://doi.org/10.1038/ismej.2013.175.

18. Adamek M, Alanjary M, Sales-Ortells $H$, Goodfellow M, Bull AT, Winkler A, Wibberg D, Kalinowski J, Ziemert N. 2018. Comparative genomics reveals phylogenetic distribution patterns of secondary metabolites in Amycolatopsis species. BMC Genomics 19:426. https://doi.org/10.1186/s12864-018 $-4809-4$.

19. Vicente C, Thibessard A, Lorenzi J-N, Benhadj M, Hôtel L, Gacemi-Kirane D, Lespinet O, Leblond P, Aigle B. 2018. Comparative genomics among closely related Streptomyces strains revealed specialized metabolite biosynthetic gene cluster diversity. Antibiotics 7:86. https://doi.org/10.3390/ antibiotics7040086.

20. Chevrette MG, Carlos-Shanley C, Louie KB, Bowen BP, Northen TR, Currie CR. 2019. Taxonomic and metabolic incongruence in the ancient genus Streptomyces. Front Microbiol 10:2170. https://doi.org/10.3389/fmicb.2019 .02170.

21. Russel J, Røder HL, Madsen JS, Burmølle M, Sørensen SJ. 2017. Antagonism correlates with metabolic similarity in diverse bacteria. Proc Natl Acad Sci U S A 114:10684-10688. https://doi.org/10.1073/pnas.1706016114.

22. Blin K, Wolf T, Chevrette MG, Lu X, Schwalen CJ, Kautsar SA, Suarez Duran HG, De Los Santos ELC, Kim HU, Nave M, Dickschat JS, Mitchell DA, Shelest E, Breitling R, Takano E, Lee SY, Weber T, Medema MH. 2017. AntiSMASH 4.0-improvements in chemistry prediction and gene cluster boundary identification. Nucleic Acids Res 45:W36-W41. https://doi.org/ 10.1093/nar/gkx319.

23. Navarro-Muñoz JC, Selem-Mojica N, Mullowney MW, Kautsar SA, Tryon JH, Parkinson El, De Los Santos ELC, Yeong M, Cruz-Morales P, Abubucker S, Roeters A, Lokhorst W, Fernandez-Guerra A, Cappelini LTD, Goering AW, Thomson RJ, Metcalf WW, Kelleher NL, Barona-Gomez F, Medema MH. 2020. A computational framework to explore large-scale biosynthetic diversity. Nat Chem Biol 16:60-68. https://doi.org/10.1038/s41589-019-0400-9.

24. Mavridou DAI, Gonzalez D, Kim W, West SA, Foster KR. 2018. Bacteria use 
collective behavior to generate diverse combat strategies. Curr Biol 28:345-355.e4. https://doi.org/10.1016/j.cub.2017.12.030.

25. Gonzalez D, Sabnis A, Foster KR, Mavridou DAl. 2018. Costs and benefits of provocation in bacterial warfare. Proc Natl Acad Sci U S A 115:7593-7598. https://doi.org/10.1073/pnas.1801028115.

26. Majeed H, Gillor O, Kerr B, Riley MA. 2011. Competitive interactions in Escherichia coli populations: the role of bacteriocins. ISME J 5:71-81. https://doi.org/10.1038/ismej.2010.90.

27. Majeed H, Lampert A, Ghazaryan L, Gillor O. 2013. The weak shall inherit: bacteriocin-mediated interactions in bacterial populations. PLoS One 8: e63837. https://doi.org/10.1371/journal.pone.0063837.

28. Mak S, Xu Y, Nodwell JR. 2014. The expression of antibiotic resistance genes in antibiotic-producing bacteria. Mol Microbiol 93:391-402. https://doi.org/ $10.1111 / \mathrm{mmi} .12689$

29. Xu Y, Vetsigian K. 2017. Phenotypic variability and community interactions of germinating Streptomyces spores. Sci Rep 7:699. https://doi.org/ 10.1038/s41598-017-00792-7.

30. Xu G, Wang J, Wang L, Tian X, Yang H, Fan K, Yang K, Tan H. 2010. Pseudo; $\gamma$-butyrolactone receptors respond to antibiotic signals to coordinate antibiotic biosynthesis. J Biol Chem 285:27440-27448. https://doi.org/10 .1074/jbc.M110.143081.

31. Nodwell JR. 2014. Are you talking to me? A possible role for $\gamma$-butyrolactones in interspecies signalling. Mol Microbiol 94:483-485. https://doi .org/10.1111/mmi.12787.

32. Rigali S, Titgemeyer F, Barends S, Mulder S, Thomae AW, Hopwood DA, van Wezel GP. 2008. Feast or famine: the global regulator DasR links nutrient stress to antibiotic production by Streptomyces. EMBO Rep 9:670-675. https://doi.org/10.1038/embor.2008.83.

33. Gubbens J, Janus M, Florea BI, Overkleeft HS, Van Wezel GP. 2012. Identification of glucose kinase-dependent and -independent pathways for carbon control of primary metabolism, development and antibiotic production in Streptomyces coelicolor by quantitative proteomics. Mol Microbiol 86:1490-1507. https://doi.org/10.1111/mmi.12072.

34. Zhang Z, Du C, de Barsy F, Liem M, Liakopoulos A, van Wezel GP, Choi YH, Claessen D, Rozen DE. 2020. Antibiotic production in Streptomyces is organized by a division of labor through terminal genomic differentiation. Sci Adv 6:eaay5781. https://doi.org/10.1126/sciadv.aay5781.

35. Granato ET, Meiller-Legrand TA, Foster KR. 2019. The evolution and ecology of bacterial warfare. Curr Biol 29:R521-R537. https://doi.org/10.1016/ j.cub.2019.04.024.

36. Kerr B, Riley MA, Feldman MW, Bohannan BJM. 2002. Local dispersal promotes biodiversity in a real-life game of rock-paper-scissors. Nature 418:171-174. https://doi.org/10.1038/nature00823.

37. Zhu H, Swierstra J, Wu C, Girard G, Choi YH, van Wamel W, Sandiford SK, van Wezel GP. 2014. Eliciting antibiotics active against the ESKAPE pathogens in a collection of actinomycetes isolated from mountain soils. Microbiology (Reading) 160:1714-1725. https://doi.org/10.1099/mic.0.078295-0.

38. Westhoff S, van Leeuwe TM, Qachach O, Zhang Z, van Wezel GP, Rozen DE. 2017. The evolution of no-cost resistance at sub-MIC concentrations of streptomycin in Streptomyces coelicolor. ISME J 11:1168-1178. https:// doi.org/10.1038/ismej.2016.194.

39. Guo YP, Zheng W, Rong XY, Huang Y. 2008. A multilocus phylogeny of the Streptomyces griseus 16S rRNA gene clade: use of multilocus sequence analysis for streptomycete systematics. Int J Syst Evol Microbiol 58:149-159. https://doi.org/10.1099/ijs.0.65224-0.

40. Schlatter D, Fubuh A, Xiao K, Hernandez D, Hobbie S, Kinkel L. 2009. Resource amendments influence density and competitive phenotypes of Streptomyces in soil. Microb Ecol 57:413-420. https://doi.org/10.1007/ s00248-008-9433-4. 